\title{
From deep inside to outer space: exploring neutron skins
}

M. Thiel ${ }^{* 1}$, S. Baunack ${ }^{1}$, D. Becker ${ }^{1}$, J. Diefenbach ${ }^{1}$, A.Esser ${ }^{1}$, M.I. Ferretti Bondy ${ }^{1}$, K.S. Kumar ${ }^{2,3}$, F.E. Maas ${ }^{1}$, H. Merkel ${ }^{1}$, U. Müller ${ }^{1}$, K.D. Paschke ${ }^{4}$, B.S. Schlimme ${ }^{1}$, C. Sfienti ${ }^{1}$, P.A. Souder ${ }^{5}$, and A. Tyukin ${ }^{1}$

${ }^{1}$ Institut für Kernphysik, Johannes Gutenberg-Universität Mainz, D-55099 Mainz, Germany

${ }^{2}$ Department of Physics, University of Massachusetts, MA 01003-9337, USA

${ }^{3}$ Department of Physics and Astronomy, Stony Brook University, NY 11794-3800, USA

${ }^{4}$ University of Virginia, Charlottesville, Virginia 22904, USA

${ }^{5}$ Syracuse University, Syracuse, New York 13244, USA

E-mail: thiel@kph.uni-mainz.de

\begin{abstract}
These days, neutron skin is one of the key words not only in nuclear physics but also in astrophysics. A variety of experimental methods has been carried out over the last years to determine the neutron skin of heavy and medium nuclei. To overcome strong model dependencies a simultaneous combination of different techniques is favoured. Combining high precision measurements using parity violating electron scattering with the opportunity to measure a multitude of nuclei given by coherent $\pi^{0}$ photoproduction allows a systematic determination across the periodic table thus benchmarking modern calculation. In a first step the systematic investigation of ${ }^{58} \mathrm{Ni},{ }^{208} \mathrm{~Pb}$ and three tin isotopes $\left({ }^{116,120,124} \mathrm{Sn}\right)$ using the latter method has been realised at the Mainzer Microtron (MAMI, Mainz). For complement, two new measuring programs using parity violating electron scattering are scheduled: within the A1 collaboration at the existing accelerator facility as well as with the future accelerator MESA within the scope of the P2 collaboration. Advantaged by the solenoidal detector design, the parity violating asymmetry for polarized electrons scattered on heavy nuclei can be determined with a $1 \%$ resolution. Status and prospects of the projects in Mainz will be discussed.
\end{abstract}

53rd International Winter Meeting on Nuclear Physics,

26-30 January 2015

Bormio, Italy

\footnotetext{
*Speaker.
} 


\section{The big picture}

Nowadays, there are still many fascinating but unresolved phenomena in all fields of physics. Whereas at first glance these open questions may seem to be separated into different subjects, a look at the big picture can help to shed light on the open issues. Such as astrophysical questions about the nature and composition of neutron stars which can be connected to questions in nuclear physics concerning the structure of stable nuclei and rare isotopes. Both cases are linked by the nuclear Equation of State (EOS) which describes the correlation between neutron-proton asymmetry, pressure, density, temperature, and energy in every nuclear system:

$$
E(\rho, \delta)=E(\rho, 0)+E_{\text {sym }}(\rho) \delta^{2}+\mathscr{O}\left(\delta^{4}\right)
$$

with the total nucleon density $\rho=\rho_{n}+\rho_{p}$ and the relative asymmetry parameter $\delta=\left(\rho_{n}-\rho_{p}\right) / \rho$. The symmetry energy $E_{\text {sym }}(\rho)$ can be estimated as the difference between the EOS of pure neutron matter and symmetric nuclear matter. Its density dependence can be characterized by the slope and curvature parameters $L$ and $K_{\text {sym }}$, respectively:

$$
E_{s y m}(\rho)=S_{v}+\frac{L}{3}\left(\frac{\rho-\rho_{0}}{\rho_{0}}\right)+\frac{K_{s y m}}{18}\left(\frac{\rho-\rho_{0}}{\rho_{0}}\right)^{2}
$$

with the symmetry energy coefficient $S_{v}=E_{\text {sym }}\left(\rho_{0}\right)$.

There is a high degree of correlation between the symmetry energy coefficient $S_{v}$ and the slope parameter $L$ whereas the neutron skin thickness $\Delta R_{n p}$ of heavy nuclei is a function of both. Due to the strong correlation between $\Delta R_{n p}$ and $L$ [1], an accurate determination of the neutron skin thickness of heavy nuclei would provide considerable constraints on the density dependence of the nuclear symmetry energy.

\section{The methods - an overview}

Due to their neutron excess, heavy nuclei are expected to have a neutron skin $\Delta R_{n p}$, which is defined as the difference between the neutron $\left(R_{n}\right)$ and proton $\left(R_{p}\right)$ root-mean-square (rms) radii. In recent years, various methods have been used to determine the neutron skin thickness of heavy nuclei and especially of ${ }^{208} \mathrm{~Pb}$. However, hadron scattering experiments involving strong probes like protons [2], antiprotons [3], and pions [4] have to rely on model calculations for the extraction of the neutron skin thickness. Moreover, measurements of giant and pygmy dipole resonance modes [5] and coherent $\pi^{0}$ photoproduction [6] can be used to determine $\Delta R_{n p}$, again depending on model predictions. To overcome the uncertainties in the model dependent interpretation of the data a direct measurement of $\Delta R_{n p}$ is essential. Parity violating electron scattering can provide the least model depend method to determine the neutron skin thickness [7]. The ${ }^{208} \mathrm{~Pb}$ Radius EXperiment (PREX) at Jefferson Laboratory (JLab) has provided the first proof-of-principle of this method [8]. However, extremly long measuring times are needed to achieve a precise determination of the neutron skin thickness with this technique.

To conclude: to determine the neutron skin of various nuclei one has to combine different methods. On the one hand, a high precision method is needed to set the absolute scale. But it is obvious, that due to the long running time such a method is not suited to scan over the entire nuclear chart. Therefore, a different approach is needed to provide the systematics over the full nuclear landscape. 


\section{Exploring neutron skins nowadays and in the future}

Aiming for a high precision measurement of the neutron skin various experiments are scheduled for the future. On the one hand, there are two approved experimental campaigns at JLab: PREX-II [9] and CREX [10]. The former one will be the follow-up measurement to PREX-I, seeking for a sensitivity of $\pm 1 \%$ for the neutron rms radius of lead, while the latter one should provide an accuracy of $\pm 0.6 \%$ in the ${ }^{48} \mathrm{Ca}$ neutron radius determination. However, both experiments will not run before 2016.

On the other hand, with the future accelerator MESA (Mainz Energy recovering Superconducting Accelerator) [11], which will be not operating before 2018, a new era of parity violating electron scattering experiments will begin in Mainz.

\subsection{P2@MESA: go for ultimate precision}

The future accelerator MESA, comprising a normal conducting injector linac and superconducting cavities in the recirculation beamline, will provide a $1.3 \mathrm{GHz}$ c.w. electron beam with energies up to $200 \mathrm{MeV}$. In the energy recovering mode (ERL mode) unpolarized electrons up to $10 \mathrm{~mA}$ will be available, whereas polarized electrons up to $300 \mu \mathrm{A}$ will be available in the external beam mode (EB mode). For the high precision parity violating electron scattering experiments within the scope of the $\mathrm{P} 2$ project, one experimental setup will be used to determine the weak mixing angle $\theta_{w}$ as well as the neutron skin thickness of ${ }^{208} \mathrm{~Pb}$. The use of a solenoid spectrometer is of particular importance for the latter one. With this concept the detectors can cover the full azimuthal range, with a peak sensitivity in the polar angular range between $21^{\circ}$ and $32^{\circ}$ for ${ }^{208} \mathrm{~Pb}$ [12].

To verify that the desired sensitivity of $0.5 \%$ for $\delta R_{n} / R_{n}$ can be achieved, calculations within this angular coverage - using a polar angle step size of $\Delta \theta=4^{\circ}$ - have been performed. Due to the high intensity electron beam, including a high degree of polarization, and the large azimuthal coverage of the detector system a total error of $\delta R_{n} / R_{n}=5.04 \cdot 10^{-3}$ (stat. $+1 \%$ sys. error), assuming a polarization degree of $85 \%$ and a dilution factor due to carbon background in the target of $10 \%$, can be reached within two month running time.

A crucial point in the determination of the parity violating asymmetry $A_{P V}$ is the separation of the ground state of ${ }^{208} \mathrm{~Pb}$ from its first excited state $(\Delta \mathrm{E} \approx 3 \mathrm{MeV})$. Hence, various magnetic field strength as well as a scan of target positions with respect to the center of the solenoid have been successfully studied within a ray trace simulation [13]. By now, the parameters of the solenoid spectrometer are finalized, allowing the determination of the neutron skin experimental setup.

Summing up, with the future accelerator MESA the neutron skin thickness of ${ }^{208} \mathrm{~Pb}$ will be determined with unprecedented precision, setting the benchmark for further neutron skin studies of medium and heavy nuclei.

\subsection{Coherent $\pi^{0}$ Photoproduction at A2}

Whereas the demand for a high precision determination of the neutron skin thickness will be satisfied in Mainz as soon as the new accelerator is avialable, the need of a systematics study is already fulfilled with the existing accelerator. The method of choice is coherent $\pi^{0}$ photoproduction $\left(\gamma+A_{\text {g.s. }} \rightarrow \pi^{0}+A_{\text {g.s. }} \rightarrow \gamma \gamma+A_{\text {g.s. }}\right)$, which represents an efficient tool to study the neutron skin 
of various nuclei, covering the whole nuclear chart range. For spin zero nuclei the coherent cross section can be expressed in the most simple plane wave impulse approximation (PWIA) [14]:

$$
\frac{d \sigma}{d \Omega} \mathrm{PWIA}=\frac{s}{m_{N}^{2}} \times \frac{1}{2} \frac{q_{\pi}^{*}}{k^{*}}\left|F_{2}\left(E_{\gamma}^{*}, \theta_{\pi}^{*}\right)\right|^{2} \sin ^{2}\left(\theta_{\pi}^{*}\right) \times A^{2} F^{2}(q) .
$$

With regard to the final state interactions of the pion on his way out of the nucleus, additional $\Delta$ inmedium effects within the framework of distorted wave impulse approximation (DWIA) are taken into account [14].

The nuclear matter form factor $F(q)$ can be extracted from the measured angular distributions and out of it the root-mean-square (rms) radii. As the charge density distributions were obtained to a high accuracy in electron scattering experiments [15], these information can be used to finally determine the neutron skin.

The A2 collaboration at the Mainz Microtron (MAMI) [16, 17] provides a detector system, comprising of the Crystal Ball [18] and TAPS [19,20] calorimeters, which is perfectly suited to detect multi-photon final states. Therefore, four different spin zero nuclei $\left({ }^{58} \mathrm{Ni},{ }^{116} \mathrm{Sn},{ }^{120} \mathrm{Sn}\right.$, and $\left.{ }^{124} \mathrm{Sn}\right)$ could be measured within three weeks of beamtime. Moreover, data with a ${ }^{208} \mathrm{~Pb}$ target was taken for a comparison to previous measurements. Here, the evolution of neutron skin along the tin isotopic chain is of special interest since most of the systematic uncertainties cancel out.

After careful calibration of all target sets, the extraction of the cross section is currently ongoing. Figure 1 shows the quality of the data for six different energy bins. The data still has to be corrected for photon flux and detection efficiency. A detailed description of the experiment and the analysis can be found in [21].
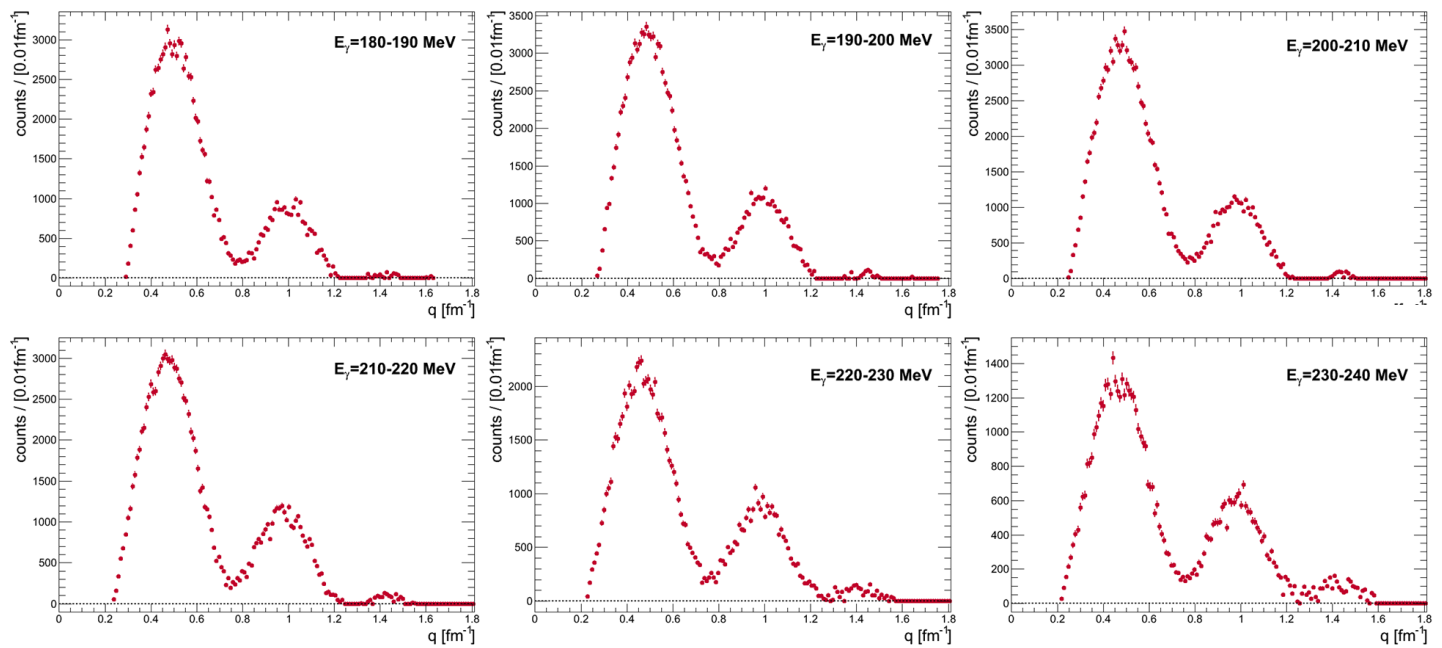

Figure 1: Diffraction pattern for the reaction ${ }^{124} \mathrm{Sn}\left(\gamma, \pi^{0}\right){ }^{124} \mathrm{Sn}$ for six different energy bins ranging from $180 \mathrm{MeV}$ to $240 \mathrm{MeV}$. 

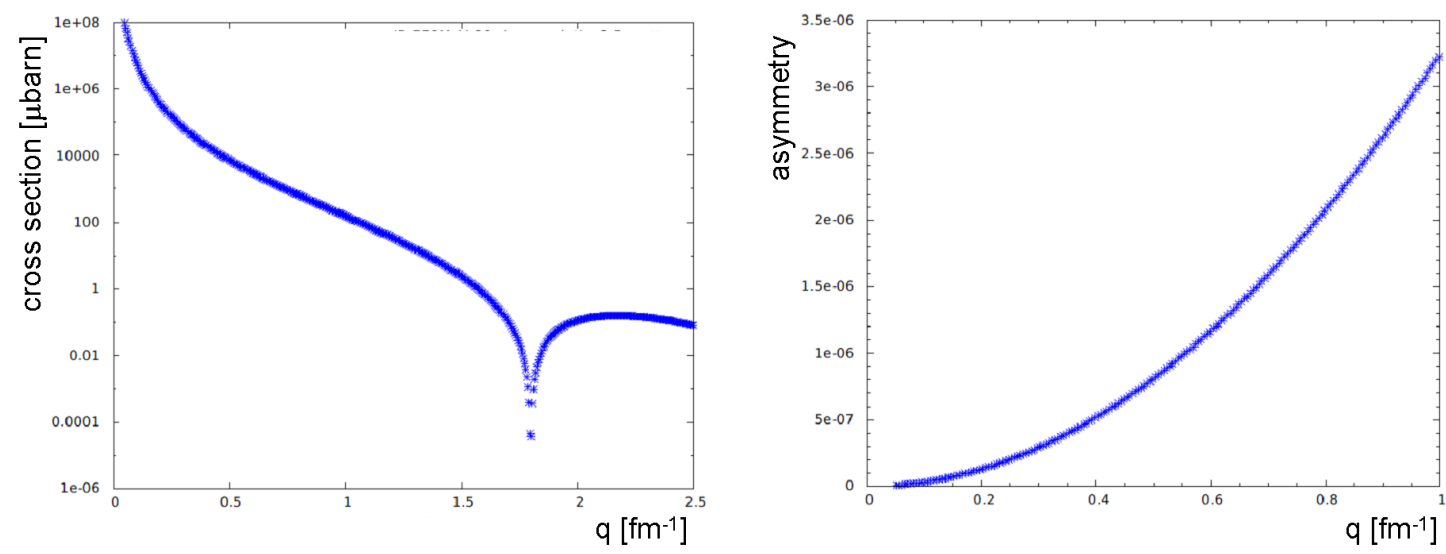

Figure 2: Left: Calculated cross section as a function of momentum transfer for electrons scattered elastically off ${ }^{12} \mathrm{C}$ at $570 \mathrm{MeV}$ beam energy. The minimal accessible angle of the spectrometers is $20^{\circ}$ which corresponds to a momentum transfer of $1.0 \mathrm{fm}^{-1}$. Right: Parity violating asymmetry $A_{P V}$ as a function of momentum transfer.

\section{Short-range program at MAMI}

As most of the high-precision experiments will start earliest in 2016, the need for a short-range program is obvious. Combining the exisiting know-how on parity violating electron scattering experiments of the A4 collaboration [22,23] with the experimental equipment of the A1 collaboration [24] provides the basis for a significant short-range program at MAMI. Whereas most of the upcoming experiments will focus on ${ }^{208} \mathrm{~Pb}$, A1@ MAMI will go for studies of ${ }^{124} \mathrm{Sn}$. Predictions from Energy Density Functionals (EDFs) [25] show a strong correlation between the neutron skin thickness of lead and tin. To verify this correlation, a measurement of tin is of importance.

As a preparatory work, longitudinally polarized electrons have been scattered elastically off an unpolarized carbon target. The main goal of this beamtime was to study the feasibility of such an experiment at the A1 spectrometer facility, investigating new beam stabilization, maximum beam current, apparatus caused false asymmetries and resulting systematic accuracy.

The choice of kinematics for this experiment was mainly set by the minimal electron beam energy at which the Møller polarimeter is still working reliably. Once the energy of the longitudinally polarized electrons was fixed to $570 \mathrm{MeV}$, the cross section as a function of the momentum transfer was calculated. Figure 2 (left) shows the diffraction pattern of the carbon cross section, illustrating the decrease of count rate with increasing momentum transfer. Due to the minimum accessible angle with the spectrometers of $\phi=20^{\circ}$ only momentum transfers above $q=1 \mathrm{fm}^{-1}$ are measurable. In contrast, the parity violating asymmetry

$$
A_{P V}=\frac{G_{F} Q^{2}}{4 \pi \alpha \sqrt{2}}\left[4 \sin ^{2}\left(\theta_{W}\right)-1+\frac{F_{n}\left(Q^{2}\right)}{F_{p}\left(Q^{2}\right)}\right]
$$

is rising with increasing momentum transfer (see figure 2, right). To optimize the experimental kinematics the product of detected rate and asymmetry $\mathrm{FOM} \propto R \times\left(A_{P V}\right)^{2}$ had to be maximized. 

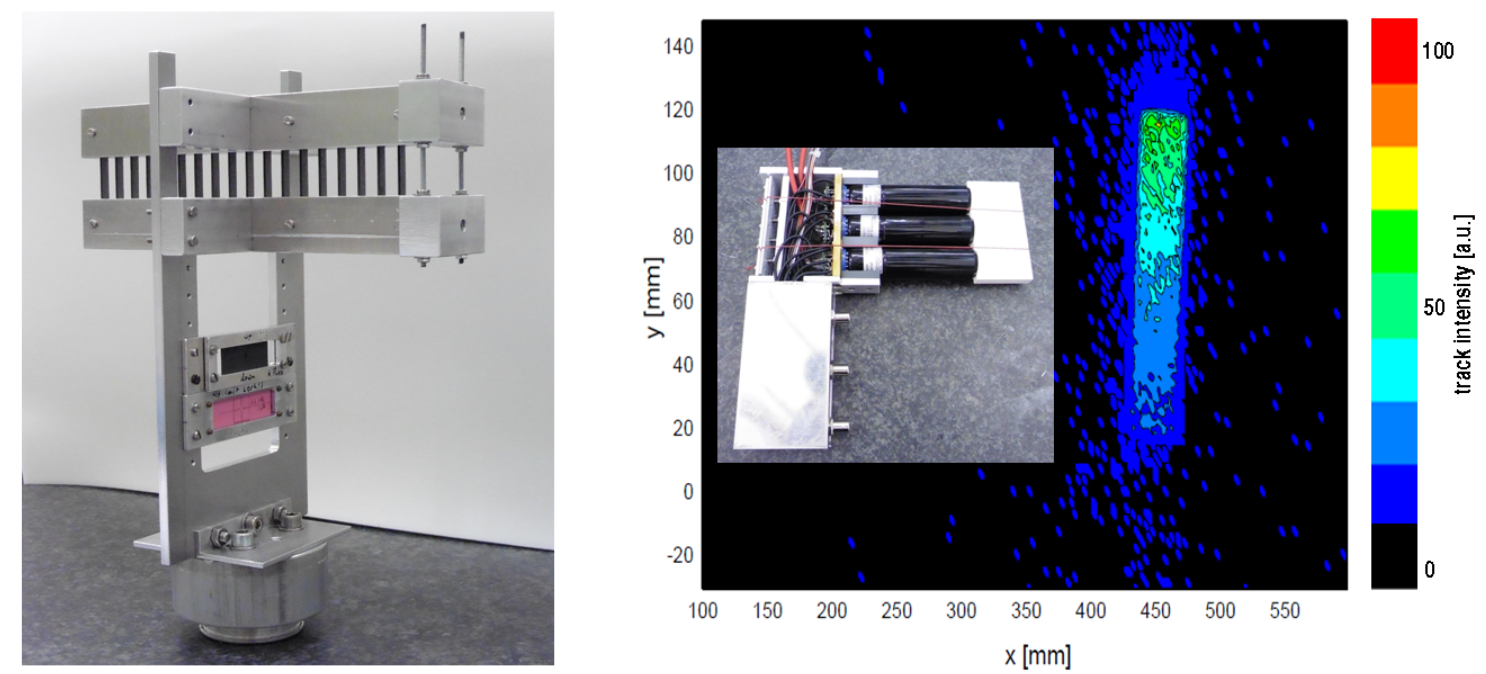

Figure 3: Left: Target ladder equipped, from top to bottom, with the extended carbon stack target, a thin carbon foil and the screen for beam positioning checks. Right: Focal plane coordinate system of the high resolution magnetic spectrometer (spectrometer A). Only coincidence events from the vertical drift chambers and the PV-detector are shown. The insert shows the PV-detector consisting of three PMTs attached to a quarz bar.

For a scattering angle of $\phi=20^{\circ}$ a parity violating asymmetry of $A_{P V} \approx 3.26 \mathrm{ppm}$ was expected. From this follows that statistical as well as systematic uncertainties had to be minimized to this level. For the former one high count rates are necessary to keep the running time reasonable. Therefore a massive carbon target was built. In total 18 target foils, each $225 \mathrm{mg} / \mathrm{cm}^{2}$ $\left(4 \times 1 \times 20 \mathrm{~mm}^{3}\right)$, were positioned over a total length of $14 \mathrm{~cm}$, able to cover the full acceptance of the spectrometer. Figure 3 (left) shows the target ladder with - from top to bottom - the extended carbon stack, a thin carbon foil $\left(45 \mathrm{mg} / \mathrm{cm}^{2}\right)$ and the screen.

To minimize the systematic uncertainties, the helicity was switched between "+" and "-" with $T=1 / f \approx 20 \mathrm{~ms}$. Pattern composed of four successive $20 \mathrm{~ms}$ periods with predefined helicity are randomly generated: "+ - + +" or "- + + -". This avoids a correlation of the asymmetry with the toggle frequency.

For the commissioning two newly build Cherenkov detectors (PV-detectors, see insert of figure 3, right), each consisting of a quartz bar $\left(100 \times 10 \times 50 \mathrm{~mm}^{3}\right)$ read out via three photomultiplier tubes (9128QSB from ET Enterprises) were installed in the focal plane of the high resolution magnet spectrometers (spectrometer A and spectrometer B). The PV-detector can be operated in two different modes: in the low current mode the full amplification range of the PMTs was exploited. Here, the PV-detectors are read out together with the spectrometer detectors. Using the information of the vertical drift chambers (VDCs), the PV-detectors could be positioned perfectly in the elastic line of the scattered electrons (see figure 3). In the high current mode - which is the experimental mode - the amplification of the PMTs is reduced to avoid a non-linear behaviour. For this mode all detector components of the spectrometer were switched off. 
The signals of the PMTs were integrated over a timewindow of $20 \mathrm{~ms}$ and read out using parts of the electronics of the former A4 experiment [26].

Furthermore, it is mandatory to monitor the beam parameters. There are in total four beam monitors: one energy monitor (ENMO), one phase intensity monitor (PIMO) and two monitors to determine the position and relative angle of the beam (XYMO) which were successfully put into operation during this experiment (figure 4, right panel). A feedback in the stabilization and monitor systems allowed the minimization of fluctuations due to helicity changes. All the information from the beam monitors as well as from the PV-detectors were combined in one data package.

Two sets of data were taken: with $\lambda / 2$-plate moved in (GVZIN) and with $\lambda / 2$-plate moved out (GVZOUT) of the laser system of the polarized source. The condition of this plate is responsible for a global polarization change of the electron beam. From this follows that data taken with GVZIN should result in a physical asymmetry with reversed sign which was considered in the analysis procedure. The parity violating asymmetry was found to be $(5.78 \pm 3.37) \mathrm{ppm}(\mathrm{GVZIN})$ and $(4.86 \pm 2.03) \mathrm{ppm}(\mathrm{GVZOUT})$ for the PV-detector placed in spectrometer A and $(-0.68 \pm 3.13)$ ppm (GVZIN) and $(2.47 \pm 2.10) \mathrm{ppm}($ GVZOUT) for the PV-detector placed in spectrometer B [27]. The results are illustrated and compared to a former measurement [28] in figure 4 (right panel). The larger discrepancy in the GVZIN data is due to the reduced statistics taken with this data set.

This first commissioning with only 2.5 days of beamtime, has successfully proven the feasibility of such type of experiments at A1. Further tests with improved setup are scheduled, especially with regard to the long-term stabilization of the beam.
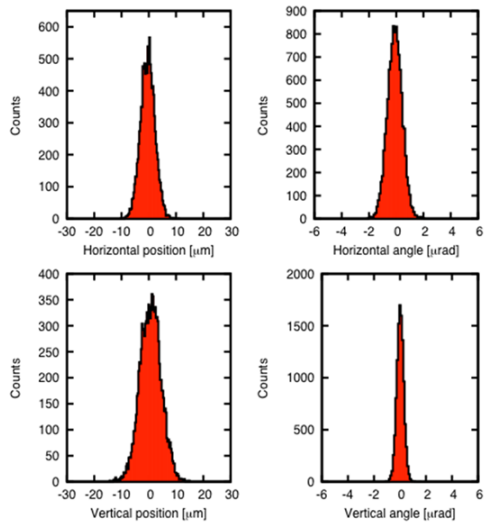
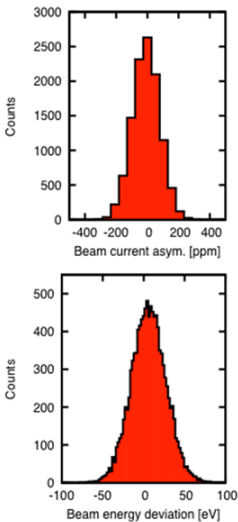

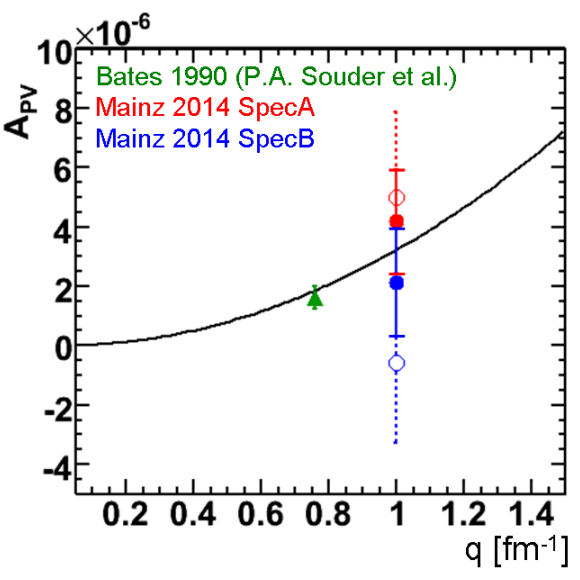

Figure 4: Left: Beam parameters after stabilization. Right: Parity violating asymmetry $A_{P V}$ for the two PVdetectors placed in spectrometer A (red points) and spectrometer B (blue points) for GVZIN (open symbols) and GVZOUT (full symbols). The results are compared to previous measurement (green point) [28] and the expected, calculated value (black line). The error bars are purely statistical. 


\section{Outlook}

After the successful test in fall last year (2014), proving the feasibility of parity violating electron scattering experiments within the environment of the A1 collaboration, a new campaign is scheduled for late summer this year, measuring the beam-normal single-spin asymmetry $A_{n}$ for ${ }^{12} \mathrm{C}$ and ${ }^{58} \mathrm{Ni}$. An improved version of the PV-detectors and the readout electronics used in the commisioning run will be used. Comparisons to theoretical calculations [29] could help to pinpoint the impact of Coulomb distortions in the range between ${ }^{12} \mathrm{C}$ and ${ }^{208} \mathrm{~Pb}$.

\section{Acknowledgments}

This work has been supported by the Deutsche Forschungsgemeinschaft through the Collaborative Research Centre SFB1044 and by the PRISMA (Precision Physics, Fundamental Interactions and Structure of Matter) Cluster of Excellence.

\section{References}

[1] X. Roca-Maza et al., Neutron Skin of ${ }^{208} \mathrm{~Pb}$, Nuclear Symmetry Energy, and the Parity Radius Experiment, Phys. Rev. Lett. 106 (2011) 252501

[2] L. Ray, W.R. Coker, and G.W. Hoffmann, Uncertainties in neutron densities determined from analysis of $0.8 \mathrm{GeV}$ polarized proton scattering from nuclei, Phys. Rev. C 18 (1978) 2641

[3] A. Trzcinska et al., Neutron Density Distributions Deduced from Antiprotonic Atoms, Phys. Rev. Lett. 87 (2001) 082501

[4] C. Garcia-Recio, J. Nieves, and E. Oset, Neutron distributions from pionic atoms, Nucl. Phys. A 547 (1992) 473

[5] A. Tamii et al., Complete Electric Dipole Response and the Neutron Skin in ${ }^{208}$ Pb, Phys. Rev. Lett. 107 (2011) 062502

[6] C.M. Tarbert et al., Neutron Skin of ${ }^{208} \mathrm{~Pb}$ from Coherent Pion Photoproduction, Phys. Rev. Lett. 112 (2014) 242502

[7] C.J. Horowitz, S.J. Pollock, P.A. Souder, and R. Michaels, Parity violating measurements of neutron densities, Phys. Rev. C 63 (2001) 025501

[8] S. Abrahamyan et al., Measurement of the Neutron Radius of ${ }^{208} \mathrm{~Pb}$ through Parity Violation in Electron Scattering, Phys. Rev. Lett. 108 (2012) 112502

[9] PREX Collaboration, PREX-II: Precision Paritiy-Violating Measurement of the Neutron Skin of Lead, Proposal to Jefferson Lab PAC38 (2011)

[10] CREX Collaboration, CREX: Parity-Violating Measurement of the Weak Charge Distribution of ${ }^{48} \mathrm{Ca}$ to 0.02 fm Accuracy, Proposal to Jefferson Lab PAC40 (2013)

[11] K. Aulenbacher et al., Opportunities for parity violating electron scattering experiments at the planned MESA facility, Hyperfine Interactions 200 (2011) 3

[12] C.J. Horowitz, private communication 
[13] M. Thiel et al., Neutron skin studies of medium and heavy nuclei, EPJ Web of Conferences 73 (2014) 07007

[14] D. Drechsel et al., Medium Effects in Coherent Pion Photo- and Electroproduction on ${ }^{4} \mathrm{He}$ and ${ }^{12} \mathrm{C}$, Nucl. Phys. A 660 (1999) 423

[15] H. de Vries, C.W. de Jager, and C. de Vries, Nuclear Charge-Density-Distribution Parameters From Elastic Electron Scattering, Atomic Data and Nuclear Data Tables 36 (1987) 495

[16] H. Herminghaus et al., The design of a cascade 800MeV normal conducting c.w. race track micrtron, Nucl. Instr. Meth. 138 (1976) 1

[17] K.-H. Kaiser et al., The $1.5 \mathrm{GeV}$ harmonic double-sided microtron at Mainz University, Nucl. Instr. Meth. A 593 (2008) 159

[18] A. Starostin et al., Measurement of $K^{-} \vec{p} \eta \Lambda$ near threshold, Phys. Rev. C 64 (2001) 055205

[19] R. Novotny, The BaF 2 photon spectrometer TAPS, IEEE Trans. Nucl. Sci 38 (1991) 379

[20] A.R. Gabler et al., Response of TAPS to monochromatic photons with energies between 45 and 790 MeV, Nucl. Instr. Meth. A 346 (1994) 168

[21] M.I. Ferretti Bondy et al., this proceeding

[22] F.E. Mass et al., Measurement of the Transverse Beam Spin Asymmetry in Elastic Electron Proton Scattering and the Inelastic Contribution to the Imaginary Part of the Two-Photon Exchange Amplitude, Phys. Rev. Lett. 94 (2005) 082001

[23] S. Baunack et al., Measurement of Strange Quark Contribution to the Vector Form Factors of the Proton at $\mathrm{Q}^{2}=0.22(\mathrm{GeV} / \mathrm{c})^{2}$, Phys. Rev. Lett. 102 (2009) 151803

[24] K.I. Blomqvist et al., The three-spectrometer facility at the Mainz Microtron MAMI, Nucl. Instr. Meth. A 403 (1998) 263

[25] J. Piekarewicz et al., Electric dipole polarizability and the neutron skin, Phys. Rev. C 85 (2012) 041302

[26] S. Baunack, The A4 experiment, Nuovo Cimento C035 (2012) 11 and references therein

[27] A. Tyukin, Entwicklung, Vorbereitung und Durchführung eines Experiments zur Paritätsverletzung an der Al Spektrometeranlage, Master Thesis, Johannes Gutenberg-Universität Mainz (2015)

[28] P.A. Souder et al., Measurement of Parity Violation in the Elastic Scattering of Polarized Electrons from ${ }^{12}$ C, Phys. Rev. Lett. 65 (1990) 6

[29] M. Gorchstein, private communication 